\title{
EL MALTRATO INFANTIL: UNA APROXIMACIÓN SOCIO- HISTÓRICA A LAS PRÁCTICAS DE CRIANZA Y MATERNIDAD \\ A LA LUZ DE LAS REPRESENTACIONES SOCIALES
}

\author{
Child abuse: a socio-historical approach to the \\ practices of parenting and motherhood in the light of \\ social representations
}

\author{
Diana Marcela Bedoya-Gallego ${ }^{1}$, Laura Carolina Jiménez Pérez ${ }^{2}$, \\ Maira Alejandra González-Gaviria ${ }^{3}$, Jhon Anthony Careth Henao ${ }^{4}$, \\ Disney Tatiana Correa Cano y León Darío Valencia Arboleda ${ }^{6}$
}

https://doi.org/10.17533/udea.rp.e339553

\section{Resumen}

El presente es un artículo de reflexión que, a la luz de la teoría de representaciones sociales, aborda analíticamente las concepciones de infancia y maternidad para plantear que, si bien las transformaciones socio-

Recibido: 2020-01-28 / Aceptado: 2021-02-13.

Para citar este artículo en APA: Bedoya-Gallego, D., Jiménez, C., González-Gaviria, M., Careth,

A., Correa, T. y Valencia, L. (2020). El maltrato infantil: una aproximación socio-histórica a las prácticas de crianza y maternidad a la luz de la teoría de representaciones sociales Revista de Psicologia Universidad de Antioquia, 13(1), e339553. doi: https://doi.org/10.17533/udea. rp.e339553. históricas de la relación entre padres e hijos han dado lugar a una modificación en las formas de manifestación y legitimación del maltrato hacia niños y niñas, no han tenido efecto en la abolición de esta lamentable 
[2] Diana Marcela Bedoya-Gallego, Laura Carolina Jiménez Pérez, Maira Alejandra González-Gaviria, Jhon Anthony Careth Henao, Disney Tatiana Correa Cano y León Darío Valencia Arboleda

práctica. Proponemos que, pese a que dichas transformaciones dieron lugar al reconocimiento del niño/a como sujeto de derechos, a la necesaria preservación de estos por parte del Estado y a la emergencia de la función materna relacionada con el afecto y el cuidado, en la sociedad contemporánea persisten creencias arraigadas que legitiman el recurso a prácticas maltratantes en la crianza de los niños y niñas.

Palabras clave: niñez, abuso infantil, tradiciones, crianza del nińo, cuidado del niño.

\section{Abstract}

This is a reflection article that, in light of the theory of social representations, analytically addresses the conceptions of childhood and motherhood to propose that, although the socio-historical transformations of the relationship between parents and children have given rise to a modification in the forms of manifestation and legitimation of the mistreatment of boys and girls, have not had an effect on the abolition of this regrettable practice. We propose that, despite the fact

that these transformations gave rise to the recognition of the child as a subject of rights, to the necessary preservation of these by the State and the emergence of the maternal role related to affection and care, in contemporary society persist beliefs that legitimize the use of abusive practices in the upbringing of children.

Keywords: childhood, child abuse, traditions, child rearing, childcare.

\section{Introducción ${ }^{7}$}

De acuerdo con Serge Moscovici (1979), principal exponente de la teoría de representaciones sociales, las representaciones que se forman y comparten entre los individuos influyen en la percepción que tienen de la realidad social, toda vez que a partir de estas adquieren saberes y construyen creencias comunes que les permiten orientarse y dominar el medio. De esta manera, la construcción de sí se liga a los procesos de interacción, identificación y pertenencia a los grupos sociales (Araya, 2002; Mora, 2002).

La representación social como forma de conocimiento contempla tres dimensiones: actitud, información y campo de representación, las cuales permiten estructurar las creencias frente a los fenómenos sociales (Araya, 2002). Así, la actitud refiere al componente afectivo o emocional de la

Esta investigación fue financiada por la Corporación Universitaria Minuto de Dios - Uniminuto (Colombia), a través de la v Convocatoria para el Desarrollo y Fortalecimiento de los Semilleros de Investigación. Expresamos nuestro agradecimiento al Semillero en Psicología Clínica. 
representación, que influencia la conducta del individuo en el medio social; este aspecto depende de la información que se adquiere y se construye a partir de la interacción con el otro y, a su vez, esta información se organiza en lo que se denomina campo de representación, de manera tal que se privilegian ciertos elementos que dan forma a la representación social.

Así pues, las representaciones sociales dan cuenta de una modalidad del conocimiento que no necesariamente se desprende del conocimiento científico ni requiere de su aprobación, ya que su elaboración depende del saber o sentido común que se comparte socialmente. Por este motivo, se convierte en un conocimiento que, aunque poco elaborado, orienta la interacción y las creencias entre los individuos (Araya, 2002; Mora, 2002; Moscovici, 1979). En efecto, como Rateau y Lo Monaco (2013) argumentan "no hacemos sino aprender la construcción ya codificada del mundo en el cual evolucionamos, los valores que lo invisten, las categorías que lo organizan y los principios mismos de su comprensión" (p. 24).

Bajo estas consideraciones, una reflexión sobre el maltrato infantil como un fenómeno cultural ligado a la crianza de los nińos y permeado por el dinamismo que las concepciones de infancia y maternidad han tenido sociohistóricamente, permite reconocer que en las diferentes épocas y culturas los grupos sociales han construido saberes compartidos relativos a creencias $y$ prácticas de crianza. En ese sentido, llama la atención que, aun cuando desde los primeros momentos del siglo xIx han surgido diferentes movimientos y tratados globales en favor de la infancia y la preservación de su bienestar (Di Gregorio, 2017; Tissera-Luna, 2018), estos no han tenido efecto en la abolición de esta lamentable práctica.

Actualmente, es una preocupación en todas las sociedades el sostenimiento y aumento de la prevalencia de este fenómeno (Organización Mundial de la Salud [oms], 2016; Instituto Nacional de Medicina Legal y Ciencias Forenses [INMLCF], 2017). Según un análisis de encuestas representativas efectuadas en 96 países sobre la prevalencia de la violencia en la nińez, se calcula que mil millones de niños/as en todo el mundo, es decir, más de la mitad de la población infantil de 2 a 17 años de edad, sufrieron violencia emocional, física o sexual en el 2016 (Organización Panamericana de la Salud [ops], 2017). Todo esto 
[4] Diana Marcela Bedoya-Gallego, Laura Carolina Jiménez Pérez, Maira Alejandra González-Gaviria, Jhon Anthony Careth Henao, Disney Tatiana Correa Cano y León Darío Valencia Arboleda

lleva a pensar, primero, que el maltrato infantil no es un fenómeno al margen de las tradiciones culturales (Rodríguez-Escobar y Rodríguez-Escobar, 2012) $y$, segundo, que es "un conflicto al que actualmente se enfrentan las diferentes disciplinas implicadas en su abordaje, puesto que no se presenta en forma aislada, sino que involucra una gran variedad de factores biopsicosociales" (Santana-Tavira, Sánchez-Ahedo y Herrera-Basto, 1998, p. 2).

En consecuencia, el presente trabajo tiene por objetivo una reflexión analítica, a la luz de la teoría de las representaciones sociales, de las concepciones de infancia y maternidad que subyacen a las prácticas de crianza que se han sostenido y avalado socialmente a lo largo del tiempo. Para ello, se hizo una revisión de fuentes primarias (Teoría de las Representaciones sociales de Moscovici, artículos de investigación, boletines, informes, encuesta nacional de demografía y salud) y secundarias (capítulos de libros y artículos de revisión), empleando los términos de búsqueda: "teoría de las representaciones sociales", "infancia" AND "historia", "prácticas de crianza" AND "historia", "cuidado infantil" AND "historia" y "maternidad" AND "historia", "maltrato infantil" "historia del maltrato infantil" "infancia" AND "maltrato. Los documentos seleccionados fueron revisados bajo el procedimiento de análisis de contenido, para ello se consideraron las categorías maltrato infantil, infancia y maternidad, a partir de las cuales se seleccionaron las unidades de análisis que permitieron dar forma a la estructura del texto. En esa vía, se recogieron los fragmentos que incluyeran la historia de la infancia, transformación de las formas de crianza a través del tiempo, la relación entre la concepción de infancia y el trato que se le ha dado a los niños, el lugar de la mujer en la sociedad y la forma como se ha vinculado al cuidado.

La información aquí presentada se deriva de la reflexión teórica llevada a cabo en la investigación Representaciones sociales del maltrato infantil en un grupo de madres estudiantes universitarias, que tuvo por objetivo conocer los significados atribuidos a los términos corrección y castigo y su relación con las representaciones que las madres partícipes del estudio tenían acerca del maltrato infantil. 


\section{Perspectiva socio-histórica de la infancia y su relación con las prácticas de crianza}

Las concepciones que históricamente se han tenido acerca de la infancia se convierten en un elemento decisivo en las prácticas de crianza que se implementan y su justificación, así como de sus componentes sociales, subjetivos e intergeneracionales, pues, si bien la crianza tiene lugar en la relación cuidador-infante, esta es "altamente sensible a las determinaciones socioculturales y al modo particular como son interpretadas en el contexto familiar” (Bocanegra, 2007, p. 203).

De acuerdo con Ariès (1988), el arte ha jugado un papel fundamental en el reconocimiento de la concepción del niño/a en las diferentes épocas. Pese a que en el siglo XIII ya existían representaciones más realistas y tiernas de la infancia, en especial, de figuras religiosas como el niño Jesús o los ángeles, hasta el siglo Xvir predominaron las representaciones artísticas de pequeños adultos. En esta época la niñez era un pasaje sin importancia, que se entendía sólo por su vínculo con la misteriosa maternidad, pero en el que la atención a las necesidades del niño/a no ocupaba el centro de atención.

Como puede deducirse de esto, las tradiciones culturales y religiosas han influido en la construcción de las representaciones de infancia y crianza. Por ejemplo, Badinter (1981) retoma los planteamientos de Cicerón y Aristóteles respecto al derecho exclusivo del padre de decidir y actuar sobre sus hijos y esposa amparado en una perspectiva de propiedad, aspecto que coincide con los fundamentos del cristianismo, en los que el hombre por derecho de creación tiene potestad sobre la mujer y los hijos (Badinter, 1981). Paralelamente, la devoción a la Virgen María, por ser la madre de Dios, ubicó a la mujer en el papel de cuidadora y dadora de vida. Sin embargo, no se desplazó la imagen sumisa y frágil de la mujer con relación al hombre, insertando al niño en la triada padre-madre-hijo, lo que le permitió a la mujer contribuir con la crianza, principalmente en lo que al amor y cuidado se refiere (Badinter, 1981).

De esta manera, a lo largo de los siglos XIV, XVII y XviII se gestó una concepción del niño/a como un ser con alma, con el que eventualmente había una transacción afectiva — referida al contacto físico — con la madre mediante 
[6] Diana Marcela Bedoya-Gallego, Laura Carolina Jiménez Pérez, Maira Alejandra González-Gaviria, Jhon Anthony Careth Henao, Disney Tatiana Correa Cano y León Darío Valencia Arboleda

la alimentación, la enseñanza de la oración y el cuidado de la higiene (Badinter, 1981; Molina, 2006). En lo que respecta al castigo, lo predominante de estos períodos era la dominación de su voluntad y comportamiento a través de la amenaza (Jaramillo, 2007; Santiago, 2007).

Las transiciones sociales experimentadas durante estos siglos, especialmente a partir del siglo XviI fueron de gran importancia para la evolución en temas relativos a la infancia. En las pinturas y fotografías familiares los niños/as, ya fueran vivos o muertos, tomaron protagonismo pues el "despilfarro demográfico", poco a poco, iba desapareciendo no sólo como resultado de las prácticas anticonceptivas, sino también porque ya los hijos iniciaron a ocupar un lugar incluso en el recuerdo (Ariès, 1988). Esto evidencia que tanto la religión como el arte influyeron significativamente en la asunción de una maternidad e infancia sensibles, dado que ofrecieron unas figuras que se constituyeron en modelos para la humanidad (Ariès, 1988; Badinter, 1981; Molina, 2006). No obstante, es sólo hasta los siglos XIX y xx que la crianza empieza a percibirse como una forma de guiar y ayudar a los niños a adaptarse a la sociedad, lo que permitió que los padres fueran más receptivos a las necesidades tanto físicas como emocionales de sus hijos, introduciendo modificaciones en las prácticas de crianza y dando lugar, por ejemplo, al diálogo (Jaramillo, 2007; Tissera-Luna, 2018). Actualmente, la crianza se halla en un marco legal que propende por amparar y garantizar la protección de los derechos de los niños/as (Izzedin y Pachajoa, 2009).

De esta manera, la crianza se concibe como un elemento fundamental en las dinámicas familiares, en donde el rol que asumen los padres en la educación de los hijos se constituye en un factor sobresaliente conformado por acciones específicas, vinculadas entre sí y susceptibles al cambio de acuerdo con el contexto social, político, económico e histórico (Bocanegra, 2007; Pulido, Castro-Osorio, Peña y Ariza-Ramírez, 2013; Santiago, 2007). Así, la concepción de infancia y prácticas de crianza han dependido de la transformación socio-histórica de unos saberes, de unas formas de pensar y actuar de los individuos en relación con el lugar otorgado al niño/a y a la relación con sus figuras de cuidado, especialmente la madre. 


\section{Maternidad: entre el rol cultural de la mujer y las necesidades de la infancia}

Lo planteado en el apartado anterior deja enunciada la alusión al cuidado y al cuidador. Históricamente, el cuidado de los niños se ha asociado a la mujer, lo cual se ha conceptualizado principalmente en el término maternidad que, a su vez, se relaciona con creencias y significados definidos por tradiciones sociales y culturales (Carbonell, 2013; Molina, 2006; Santelices, Farkas, Montoya, Galleguillos, Carvacho, Fernández, Morales, Taboada y Himmel, 2015; Palomar, 2005). Un breve repaso por algunos periodos de la historia hace evidente que esta relación entre ser mujer y ser madre surge por la doble representación que hay de la mujer como dependiente y autónoma, la cual tiene origen —entre otras - en las creencias de la cultura griega y en la tradición judeo-cristiana.

En la cultura griega las diosas representaban las cualidades femeninas, así las vírgenes representaban atributos de independencia y autosuficiencia, mientras que las vulnerables, al ser dominadas y humilladas, se constituían como esposas, madres o hijas que dependían de la vinculación que establecían desde estos roles (Badinter, 1981; Molina, 2006). Por su parte, en la tradición judeo-cristiana, la imagen de Eva contribuyó a que la mujer fuera representada como débil, por lo cual merecía ser dominada por el hombre; pero, esta representación fue revalorizada mediante la figura de la Virgen María por su devoción a la familia (Molina, 2006). Por consiguiente, las interpretaciones bíblicas de Eva y la Virgen María — la primera asociada al pecado y la segunda a la ternura y el cuidadocontribuyeron a que durante la Edad Media se instituyera un rol femenino vulnerable y dependiente, lo que implicó que a la mujer se le encargaran tareas, para ese entonces, poco valoradas como el cuidado de los hijos. Esto se debía a que se consideraba que tenía capacidad de brindar un amor carnal y pasional importante para la supervivencia biológica durante los primeros años y enseñar el control del comportamiento moral al niño/a, mientras que el hombre se encargaba de la educación intelectual (Badinter, 1981; Molina, 2006).

Más adelante, en la era romántica, surgieron modificaciones sobre las formas de ejercer la maternidad que permiten entender que también 
[8] Diana Marcela Bedoya-Gallego, Laura Carolina Jiménez Pérez, Maira Alejandra González-Gaviria, Jhon Anthony Careth Henao, Disney Tatiana Correa Cano y León Darío Valencia Arboleda

se relacionó con las diferencias que establecían las clases sociales (Badinter, 1981; Molina, 2006). En las clases pobre y aristócrata la maternidad no tenía lugar, en la primera, porque para contribuir al sustento de la familia estas mujeres eran quienes cuidaban de los niños de las familias aristócratas que tenían descendencia y, en la segunda, por el privilegio del cultivo del intelecto y las relaciones sociales, de manera que, las mujeres de esta clase se dedicaron al aprendizaje de la filosofía, de los negocios, a leer y escribir. Es en la clase burguesa que la maternidad tiene su ascenso pues, por lo menos durante este periodo esta fue entendida como el objetivo central en la vida de la mujer de esta clase, dado que "no tenía más preocupaciones y temas de conversación que sus hijos" (Badinter, 1981, p. 179). Esto implicaba que acogieran de manera instintiva y natural el cuidado de los niños, reconocidos como inocentes y necesitados de protección (Badinter, 1981, p. 182), sin embargo, las madres burguesas no tenían la potestad de definir los criterios de crianza, responsabilidad que atañía a los padres, la iglesia y la comunidad (Badinter, 1981; Molina, 2006).

De esta manera, las tradiciones religiosas y sociales de Europa permearon significativamente los criterios de crianza de cada época, a lo que se asociaba una concepción contradictoria del nińo/a como inocente y atravesado por la maldad, lo que justificaba la recurrencia a prácticas que, reprimiendo su conducta, favorecieran el ajuste a lo socialmente deseado (Vindas, 2010). Es relevante mencionar que la identificación maternidad-crianza, que tuvo mayor fuerza en el siglo xIx, contribuyó a que no se alentara más el castigo físico, toda vez que esta relación ya no se orientaba sólo a lo instrumental, de modo que el afecto empezó a ocupar un lugar (Molina, 2006). Posteriormente, en los comienzos del siglo xx, se instruye a la mujer para desempeñarse en la crianza de los hijos, pues su responsabilidad está en proporcionar apoyo moral y emocional a los miembros de su familia para favorecer así el desarrollo del niño y, por lo tanto, el bienestar social (Molina, 2006; Vindas, 2010) — aspecto que además tuvo efecto en que la maternidad se convirtiera en un asunto tanto privado como público (Palomar, 2005)—. 

maternidad a la luz de las representaciones sociales

De lo dicho hasta ahora se colige que la maternidad, entendida como una práctica en cambio, se modifica conforme las transformaciones del contexto, en ese sentido, "ya no puede verse como un hecho natural, atemporal y universal, sino como una parte de la cultura en evolución continua" (Palomar, 2005, p. 40). Está determinada tanto por condiciones individuales como por la propia experiencia de la madre en su condición de hija durante la niñez, lo que sugiere una transmisión intergeneracional de este comportamiento (Vindas, 2010), así como por aspectos tradicionales, costumbristas y normativos que influyen en este quehacer (Carbonell, 2013; Lerner, 1993; Molina, 2006; Palomar, 2005; Vindas, 2010).

Todo esto permite cuestionar aquello que se concibe como maternidad y la finalidad de esta. En primera instancia, se considera pertinente recurrir a Molina (2006), pues de manera sintética plantea que la maternidad comprende diversas funciones como "procreación, nutrición, corrección conductual, formación moral, formación afectiva, instrucción, por nombrar algunos, los cuales no siempre han sido adjudicados a la madre a lo largo de la historia” (p. 102). En segunda instancia, a Camacho (como se citó en Vindas, 2010), quien define el maternaje $e^{8}$ como:

Todas las tareas de cuidado desarrolladas por las mujeres alrededor de las hijas e hijos, sean estos biológicas/os o adoptivos; concepción tradicional de la labor que desempeñan las mujeres en la reproducción, crianza, educación, cuidado de los hijos e hijas (p. 48).

Así pues, el proceso de crianza cumple con unos objetivos más o menos comunes dado que, por un lado, favorece el proceso de desarrollo físico y psíquico y, por el otro, la capacidad del niño para alcanzar un estado de felicidad y seguridad de sí mismo y del mundo; siendo estos dos aspectos fundamentales para que se involucre, cuestione, tome decisiones, reflexione y haga solicitudes a nivel social (Vindas, 2010). De esta manera, la crianza, generalmente asociada a la madre, implica para esta una experiencia de evocación de vivencias previas y comunes, que se convierten en referente

\footnotetext{
8 Recamier (1979) propuso el uso de este concepto para referirse al conjunto de procesos físicos, psíquicos y afectivos que se desarrollan y se integran durante la maternidad.
} 
[10] Diana Marcela Bedoya-Gallego, Laura Carolina Jiménez Pérez, Maira Alejandra González-Gaviria, Jhon Anthony Careth Henao, Disney Tatiana Correa Cano y León Darío Valencia Arboleda

para el cuidado y protección que debe proveer al infante. A propósito de este cuidado, actualmente se considera que debe caracterizarse por ser sensible, es decir, ajustado a las señales y comunicaciones del niño/a, tanto físicas como psicológicas (Carbonell, 2013; Santelices et al., 2015; Torres, 2006).

Ahora bien, las ideas en torno al origen del comportamiento materno permiten fundamentar que este no es innato o biológico, se forja a través de la socialización que la madre tiene, ya sea a nivel familiar, cultural o con su hijo (Badinter, 1981; Palomar, 2005). Este aspecto lleva a desmitificar la maternidad idealizada, pues lejos de la perfección:

El maternaje, teñido de dudas e imposibilidad de reproducción exacta de roles aprendidos, se convierte en una tarea de construcción diaria, en un espacio de experimentación cotidiana, en donde, como se enunció, la construcción y la reflexión en espacios socioeducativos se vuelve fundamental (Vindas, 2010, p. 50).

Esta propuesta permite pensar que la maternidad en la actualidad no se sujeta a la autoridad tradicional, por lo tanto, la madre puede elegir entre múltiples formas de proceder, lo cual le da cierto alivio frente a las altas y casi ilimitadas responsabilidades de cuidado y crianza que se le demandan (Gilligan; 2013; Molina, 2006; Vindas, 2010). No obstante, esta idea parece ser una utopía, toda vez que frente al quehacer materno continúan existiendo mandatos provenientes de la familia que tradicionalmente la mujer ha obedecido, situación que en muchos casos la inserta en una doble lógica de comportamientos.

A modo de ejemplo, basta con considerar la transformación que como sociedad hemos experimentado al respecto, que ha favorecido la participación de la mujer en ámbitos diferentes al hogar como lo son el educativo y el laboral, transformando así la experiencia de feminidad y permitiendo que las mujeres encuentren múltiples maneras de alcanzar su realización personal (Martínez, 2015). Esta situación también transforma el quehacer materno con el tiempo, pues se relaciona con condiciones propias de la sociedad y la cultura, como lo son las legales, económicas y políticas, entre otras, que definen dicho rol (Badinter, 1981; Esquivel, 2013; Gilligan, 2013; Lerner, 1993). Al respecto Aranda (2017) señala que 
Desde la infancia [las mujeres] son socializadas en actitudes de cooperación, intimidad y emocionalidad, mientras que al descubrir el mundo público de lo académico se da cuenta de que debe asumir actitudes de la subjetividad masculina (objetividad, neutralidad, desapego, etc.) para atravesar por él y alcanzar logros reconocidos (p.149).

De esta manera, culturalmente se asume que la mujer debe cumplir con funciones que tradicionalmente se han asociado a una lógica masculina, así como con aquellas que se ajustan a dichos roles y son aprobadas o bien vistas socialmente para las mujeres, por tanto, debe adquirir capacidades contradictorias para asumir su rol (Aranda, 2017). Todo esto la expone a condiciones de riesgo (v. gr. estrés, depresión) que redundan en efectos negativos, tanto en su propia imagen como en el ejercicio de la maternidad, máxime cuando carece de una red de apoyo sólida.

En este momento se hace pertinente proponer que, si bien las funciones relativas a esta práctica comúnmente se han asociado a la mujer, la maternidad no es una responsabilidad exclusiva de esta, ya que no es una cuestión biológica (Badinter, 1981; Lerner, 1993) y, aun cuando la cultura haya sostenido esta idea a lo largo del tiempo y lo continúe haciendo (Esquivel, 2013; Lerner, 1993), tanto la madre como el padre conforman una unidad cuyo objetivo es la crianza de los hijos en un ambiente cálido, de estimulación e interacción. De este modo, puede desplazarse tanto el término de maternidad como el de paternidad al de parentalidad (Palomar, 2005); concepto que destaca que la crianza y el cuidado de los hijos es una tarea en común dentro del hogar, sin distinción de sexo o género (Tronto, 1987).

Lo problemático radica en que nuestra cultura continúa tendiendo hacia la creencia de que la madre, como figura femenina, es la más capacitada para desempeñar el rol de cuidador principal de los niños/as, lo que además se ha asociado a la posesión de un rol definido y a la valoración como mujeres (Lerner, 1993; Tronto, 1987). Esta situación no sólo genera que la mujer siga siendo la encargada de propender por la crianza de los niños pese a las demandas sociales y cargas laborales adicionales (Esquivel, 2013; Flores y Tena, 2014; Gilligan, 2013; Tronto, 1987), sino que se convierte en una limitación para el ejercicio activo de la paternidad (Aguayo, Levtov, Barker, Brown y Barindelli, 2017). 
[12] Diana Marcela Bedoya-Gallego, Laura Carolina Jiménez Pérez, Maira Alejandra González-Gaviria, Jhon Anthony Careth Henao, Disney Tatiana Correa Cano y León Darío Valencia Arboleda

Estas contradicciones, poco a poco, han dado lugar a movimientos de carácter principalmente feminista, que propenden por la desestigmatización de la mujer y el cuestionamiento de los discursos normalizadores de los roles asignados a esta, fenómeno que se suma a los argumentos que sostienen el dinamismo de las sociedades y sus producciones culturales y, con ello, las transformaciones que sufren las creencias y comportamientos como fruto de los saberes y actitudes de los individuos y colectivos (Lerner, 1993). Pese a ello, la prevalencia del maltrato infantil se sostiene, dando lugar al siguiente planteamiento: las transformaciones en las concepciones de infancia y maternidad han dado lugar a una modificación en las formas de manifestación y legitimación del maltrato hacia niños y niñas, sin embargo, sus implicaciones han sido menores en la erradicación de este fenómeno.

\section{Reconocimiento del maltrato infantil como una práctica arraigada a tradiciones culturales}

Hasta aquí se ha develado un panorama de la crianza insertado en la cultura y en las formas como históricamente se ha concebido al niño/a y a la maternidad, puesto que las representaciones sociales que se han tenido de estos han estado inmersas en la manifestación de maltrato infantil. Las agresiones contra los niños/as han existido desde tiempos remotos apoyadas en justificaciones de diferente índole, lo cual ha hecho ver estos actos como una expresión de la sociedad de la época. Santana-Tavira et al. (1998) señalan que, a lo largo de la historia, a los niños se les ha sacrificado para agradar a los dioses, mejorar y preservar la especie e incluso para disciplinar. Como ejemplo de esto se encuentran referencias mitológicas tales como la de Medea, quien mata a sus dos hijos para vengarse de Jasón, o Saturno, quien devora a su hijo. Asimismo, esta postura se encuentra consignada en expresiones aristotélicas del orden de: "un hijo o un esclavo son propiedad, y nada de lo que se hace con la propiedad es injusto" (Santana-Tavira et al., 1998, p. 2). De esta manera, el asesinar a los nińos era algo natural, permitido y aceptado, incluso en el contexto nazi todo niño con defectos físicos era asesinado en pro de conservar la pureza de la raza alemana y, en China, surgieron rigurosos mecanismos de control natal (Santana-Tavira et al., 1998). 
Ahora bien, a partir del siglo XIX en Europa y Estados Unidos, a raíz de ciertos acontecimientos se empieza a centrar la atención en la niñez. En Europa, por ejemplo, la industrialización, la urbanización, la migración, el movimiento feminista y los pensadores liberales generaron inquietud frente a ciertos fenómenos sociales que estaban afectando de manera significativa a los individuos, a la vez que los invitaban a tener una conciencia de responsabilidad frente a la sociedad. Uno de los hechos importantes para la época fue la explotación de los niños en el ámbito laboral, aspecto que llamó la atención de Marx (como se citó en Carli, 1994) quien lo relacionó con el concepto de familia, que para él variaba según las diferentes clases sociales y, a su vez, se encontraba relacionado con el concepto de infancia.

Esta ligazón entre clase social y concepción de familia (Badinter, 1981) influye en las diferentes formas de vinculación que se gestan al interior de las familias, un ejemplo de ello es la relación social y política de la época en la cual los niños hacían parte del capital de trabajo proletario, motivo por el cual Marx divulga y se opone a "las violencias más profundas del capitalismo industrial, la fragmentación social de la infancia, la explotación del trabajo infantil y su dignificación en un orden comunista" (como se citó en Carli, 1994, p. 6), denunciando así la explotación laboral infantil a cargo de los padres en familias de clase baja, situación que se presentaba a causa de las condiciones de trabajo del proletariado.

Por otra parte, Santana-Tavira et al. (1998) refieren que en 1871 en Nueva York (Estados Unidos) surge la primera Sociedad para la Prevención de la Crueldad con los Niños como una respuesta a la demanda de los ciudadanos que buscaban proteger los derechos de los menores de edad que eran maltratados puesto que, para el momento, no existía una instancia legal que los protegiera, a pesar que desde 1868 el médico Ambrosie Tardieu había descrito por primera vez casos referidos a niños/as golpeados. Así pues, la preocupación por el bienestar físico de los menores de edad en términos que impliquen aspectos legales e institucionales inicia en el ámbito médico. Ya en 1961, un grupo de médicos encabezado por Henry Kempe retoman el término sindrome del niño golpeado para establecer a partir de este una categoría clínica en la que se hace referencia a casos de niños internados en las clínicas 
[14] Diana Marcela Bedoya-Gallego, Laura Carolina Jiménez Pérez, Maira Alejandra González-Gaviria, Jhon Anthony Careth Henao, Disney Tatiana Correa Cano y León Darío Valencia Arboleda

debido a situaciones de negligencia y golpes físicos provocados por sus padres, develando una situación de interés social y político que se convirtió en el foco de atención, abarcando más adelante el abuso sexual y el maltrato psicológico (Grinberg, 2015).

Respecto a la ampliación del concepto de maltrato infantil, Fontana indica que "estos niños podían ser agredidos no solo en forma física sino también emocionalmente o por negligencia" (como lo citó Santana-Tavira et al., 1998, p. 2), reemplazando así la expresión golpeado por maltratado, dando paso al concepto que conocemos actualmente. Debido a esto, este fenómeno empieza a reconocerse como una problemática social y a suscitar el interés por el buen trato hacia los niños. A finales del siglo xIx en Europa y Estados Unidos, estos malos tratos empezaron a ser sancionados moral y legalmente por el Estado y la sociedad. Grinberg (2015) refiere que sólo a partir de los ańos sesenta el fenómeno del maltrato infantil cobra fuerza como un asunto que merece atención y, para los años 80, el concepto de maltrato infantil se había extendido "a los países anglosajones, Europa y el resto del mundo sucesivamente" (p. 79).

En este sentido, poner en evidencia a nivel social las necesidades del niño y lograr el reconocimiento del maltrato dio lugar al desarrollo de manifiestos políticos y gubernamentales que procuraban la preservación del bienestar de los menores de edad, dado que adquieren un papel central para el Estado y la sociedad en general (Di Gregorio, 2017; Jaramillo, 2007). Cabe señalar que, en la base de estos manifiestos, se encuentran los movimientos de reforma de la niñez que, a mediados del siglo XIX, empiezan a defender una concepción de la niñez mediante la cual:

Los niños y niñas eran considerados inherentemente dependientes, indefensos y necesitados de la protección de los adultos, y la niñez era construida como inocente y natural, como una etapa de la vida especial, despreocupada y definida por la negativa en relación a la adultez, que debía ser protegida de las dificultades del "mundo adulto" (Tissera-Luna, 2018, p. 43).

Concepción que se ha sostenido en el tiempo, continuando vigente en nuestra época, y que ha permitido, primero, sustentar aquellas prácticas mediante las que se promueve la protección de la infancia y, segundo, evidenciar 
que los parámetros respecto ella, en torno a los cuales giran los movimientos que buscan proteger a los niños, excluyen prácticas culturales que relativizan la niñez. En esa medida, la infancia puede ser vivida de otras maneras toda vez que depende del contexto histórico, social y cultural (Tissera-Luna, 2018).

Ahora bien, respecto a estos movimientos que promueven la protección de la niñez es importante resaltar que, sólo después de un siglo de su aparición, es que toman una verdadera presencia a nivel mundial, pues hasta 1959 (año en el que la Organización de Naciones Unidas onu reconoce la Declaración de los Derechos del Niño) y 1989 (año en el que tiene lugar la Convención Internacional de los Derechos del Nińo CDN) los diferentes movimientos tenían un reconocimiento e impacto sectorizado, ya fuera en el país o países de origen. Así, la participación de diferentes países en lo relacionado con el reconocimiento, la prevención y atención de las problemáticas que afectan a la niñez, así como la promoción del bienestar durante la infancia, tomó un mayor tiempo en establecerse (Tissera-Luna, 2018).

Para aproximarse a la concepción de maltrato infantil basta recurrir a la definición más empleada, ofrecida por la oms (2016), mediante la cual se entiende que este hace referencia a:

Los abusos y la desatención de que son objeto los menores de 18 años, e incluye todos los tipos de maltrato físico o psicológico, abuso sexual, desatención, negligencia y explotación comercial o de otro tipo que causen o puedan causar un daño a la salud, desarrollo o dignidad del niño, o poner en peligro su supervivencia, en el contexto de una relación de responsabilidad, confianza o poder. La exposición a la violencia de pareja también se incluye a veces entre las formas de maltrato infantil (párr. 2).

Sin embargo, cabe aclarar que se pueden presentar ciertas variaciones en la definición dada por las diferentes instituciones y organizaciones interesadas en el bienestar de la infancia. Así mismo, se encuentra que la clasificación del maltrato infantil es diversa; dentro de la más reconocida se encuentra el maltrato físico, el psicológico, el emocional, la negligencia y el abuso sexual.

Una de las primeras referencias al maltrato físico se encuentra en Kempe, Silverman, Steele, Droegemueller y Silver (1962), quienes lo presentaron como el uso abusivo de la fuerza física por parte de padres o parientes para herir o 
[16] Diana Marcela Bedoya-Gallego, Laura Carolina Jiménez Pérez, Maira Alejandra González-Gaviria, Jhon Anthony Careth Henao, Disney Tatiana Correa Cano y León Darío Valencia Arboleda

lesionar gravemente a un niño/a. Con relación al maltrato emocional, O’Hagan (1995) plantea que consiste en la exposición repetida de los niños a situaciones de miedo, humillación, angustia o desesperación; este afecta su capacidad de experimentar y regular emociones positivamente, así como su desarrollo social. Por su parte, el maltrato psicológico implica un comportamiento repetitivo por parte del cuidador que afectan la maduración de facultades mentales en el niño/a (inteligencia, percepción, atención, reconocimiento y memoria) y su desarrollo moral, desencadenando consecuencias a nivel social, emocional y educativo (O’Hagan, 1995). Respecto a la negligencia, Palacios, Moreno y Jiménez (2014) la definen como toda "situación en la que las necesidades físicas básicas de un niño/a y su seguridad no son atendidas por quienes tienen la responsabilidad de cuidarlo" (p. 12). A su vez, estos autores definen el abuso sexual y dicen que es la "utilización que un adulto hace de un menor de 18 años para satisfacer deseos sexuales” (p. 13).

De esta manera, el maltrato infantil, asumido como una forma incorrecta de concebir la crianza, queda inscrito en un marco legal a partir del cual se hace posible reconocer y respetar al niño/a sus derechos (Izzedin y Pachajoa, 2009). En este mismo sentido, Posada, Gómez y Ramírez (2008) plantean que una crianza humanizada sintoniza con los derechos de los niño/as y adolescentes, toda vez que no se basa en el autoritarismo y el control sino en la reafirmación de la voluntad del menor y en su capacidad de decisión.

Ahora bien, con el panorama que se ha expuesto acerca del maltrato infantil a la luz de las transformaciones que han tenido las concepciones de infancia y maternidad y su consecuente relación con la crianza se vislumbra una doble lógica. Por un lado, la determinación de instituciones públicas y privadas de preservar la vida y el bienestar de los menores de edad impacta en la formulación de políticas públicas en favor de este interés, así como en el diseño e implementación de programas de promoción, prevención e intervención que procuran el desarrollo de competencias parentales positivas. Por otro lado, la persistencia de creencias y prácticas que, amparadas en representaciones sociales acerca de la relación entre padres e hijos, legitiman acciones que no favorecen el adecuado desarrollo de los nińos/as; al respecto Carreño y Rey (2010) afirman que: 
El mismo término maltrato es un desafío a esa cultura, que continúa construyendo discursos sobre las relaciones entre el adulto y el niño basadas en los criterios de la carencia, de lo que todavía no se es, teniendo como patrón de medida al adulto (p. 810).

Esto plantea una condición de asimetría en las relaciones entre adultos y niños/as que se ha malinterpretado, dando lugar a que la necesidad del niño/a de contar con una familia a partir de la cual pueda configurarse como un sujeto con identidad y autonomía mediante una relación saludable con el otro, corra el riesgo de tergiversarse bajo la premisa de la dependencia del niño/a al adulto (Sünker y Moran-Ellis, 2018). Máxime si sus cuidadores primarios se encuentran expuestos a factores de riesgo individuales o contextuales, de tal suerte que acciones maltratantes se solapen en prácticas de corrección y castigo. En suma, el maltrato infantil es un fenómeno que trasciende lo individual y se inserta en la cultura a través de las formas de representar la realidad y de vincularse con el otro.

\section{Conclusión}

El recorrido histórico realizado permitió identificar que se pasó del no reconocimiento del niño como sujeto de derechos al esfuerzo político de su visibilización; esta transición puso en evidencia que se han aceptado e incluso aún hoy se avalan socialmente ciertas prácticas de crianza que podrían enmarcarse como concepciones asociadas al maltrato infantil. Así, se encuentra que los estilos de crianza están influenciados por la estructura social de la época, lo que a su vez implica unas construcciones culturales, simbólicas, lingüísticas, respecto de la crianza, la infancia, la maternidad y la paternidad, dejando manifiesta una relación inexorable entre el individuo y la cultura de la cual participa, entre la relación cuidador-infante y los discursos culturales que la penetran.

De esta reflexión emergieron dos ideas centrales. La primera, relativa a cómo se ha entendido históricamente al niño, encontrando que, aunque en la actualidad la protección de la niñez se halla legalmente constituida como un deber y responsabilidad del Estado, la familia y 
[18] Diana Marcela Bedoya-Gallego, Laura Carolina Jiménez Pérez, Maira Alejandra González-Gaviria, Jhon Anthony Careth Henao, Disney Tatiana Correa Cano y León Darío Valencia Arboleda

la comunidad en general (Carbonell, 2013; Izzedin y Pachajoa, 2009), persiste una dificultad para comprender y percibir al niño como un sujeto de derechos, haciendo uso de discursos adulto-céntricos que no le permiten ejercer su autonomía y que no siempre atienden a una crianza basada en las necesidades que se configuran según el curso vital (Carbonell, 2013; Carreño y Rey, 2010). La segunda, relacionada con las funciones de la maternidad — que entre otras cosas implican la crianza y el cuidado- , asociadas a la mujer como principal cuidadora, mediante las cuales se sostienen discursos normalizadores de la relación mujer-maternidad.

A partir de estas ideas surgen cuestionamientos acerca del carácter vinculante que está implicado en las prácticas de crianza, entre ellos el lazo que se establece entre el niño y la madre. Esta continúa siendo el principal cuidador, tanto de los aspectos físicos como afectivos de sus hijos (Esquivel, 2013; Flores y Tena, 2014; Gilligan, 2013; Tronto, 1987), lo que en conjunto con los nuevos roles que actualmente enfrenta se convierte en un factor de riesgo para sus actitudes maternales y puede llegar a suponer afecciones en su salud mental. Este malestar se puede expresar en altos niveles de estrés, agotamiento y frustración respecto a su proyecto de vida que encuentra como vía de descarga la reactividad hostil hacia sus hijos (Aranda, 2017), fundamentalmente, cuando se carece de una red de apoyo sólida.

Finalmente, si se reconoce que las consecuencias que se han documentado respecto del maltrato infantil señalan efectos tanto a nivel personal como social, es decir, retrasos en el desarrollo cognitivo, físico, emocional del niño/a, así como retrasos en el desarrollo social, económico y político de un país, se hace necesario continuar con el esfuerzo por desarrollar estudios y programas que no se limiten solamente a la atención de los niños, sino que también tomen en cuenta perspectivas que contemplen las relaciones sociales que se han construido y transmitido de generación en generación al interior de la familia. En efecto, estas han sido atravesadas por cuestiones políticas y económicas (Sünker y Moran-Ellis, 2018), por lo que se pone en evidencia la necesidad de 
penetrar las estructuras sociales que históricamente han sostenido y avalado formas de crianza que afectan el sano desarrollo de los menores de edad. Entre estas estructuras se resalta de manera especial la familia, pues es importante continuar pensando el papel que ha tenido, no sólo en el sostenimiento de prácticas que afectan el desarrollo, sino también en la invención de nuevas formas de relación entre sus miembros; esto sin duda permitirá entender y promover los elementos adecuados para crear bases familiares sólidas en las que el buen trato, el aprendizaje bidireccional y la corresponsabilidad estén presentes.

\section{Referencias}

Aguayo, F., Levtov, R., Barker, G., Brown, V. y Barindelli, F. (2017). Estado de la paternidad en América Latina y el Caribe. doi: 10.13140/RG.2.2.17120.28168.

Aranda, N. (2017). Imágenes y perspectivas sobre la maternidad en las estudiantes de la Universidad Nacional de Santiago del Estero. Revista Latino-Americana de Geografia e Género, 8(1), 145-159. doi: 10.5212/Rlagg.v.8.i1.0009.

Araya, S. (2002). Las representaciones sociales: Ejes teóricos para su discusión. San José, Costa Rica: Facultad Latinoamericana de Ciencias Sociales (FLACSO).

Ariès, P. (1988). El niño y la vida familiar en el antiguo régimen. Barcelona: Taurus.

Badinter, E. (1981). ¿Existe el amor maternal? Historia del amor maternal. Siglos XVII al XX. Barcelona, España: Paidós.

Bocanegra, E. (2007). Las prácticas de crianza entre la Colonia y la Independencia de Colombia: los discursos que las enuncian y las hacen visibles. Revista Latinoamericana de Ciencias Sociales, Niñez y Juventud, 5(1), 201-232. Recuperado de http://www.scielo.org.co/scielo.php?script=sci_arttext\&pid= S1692-715X2007000100007.

Carbonell, O. (2013). La sensibilidad del cuidador y su importancia para promover un cuidado de calidad en la primera infancia. Ciencias Psicológicas, 7(2), 201207. Recuperado de http://www.scielo.edu.uy/pdf/cp/v7n2/v7n2a08.pdf.

Carli, S. (1994). Historia de la infancia: una mirada a la relación entre cultura, educación, sociedad y política en Argentina. Revista del Instituto de Investigaciones en Ciencias de la Educación, (4), 3-11. Recuperado de http://repositorio.filo. uba.ar/bitstream/handle/filodigital/4900/CArli.pdf?sequence=1 \&isAllowed=y. 
[20] Diana Marcela Bedoya-Gallego, Laura Carolina Jiménez Pérez, Maira Alejandra González-Gaviria, Jhon Anthony Careth Henao, Disney Tatiana Correa Cano y León Darío Valencia Arboleda

Carreño, C. y Rey, A. (2010). Reflexiones en torno a la comprensión del maltrato infantil. Universitas Psychologica, 9(3), 807-822. Recuperado de http://www. scielo.org.co/pdf/rups/v9n3/v9n3a16.pdf.

Di Gregorio, N. (2017). Prácticas de crianza en la primera infancia. En Pereira, P. (Coord.). Reflexiones sobre las pautas de crianza de los niños y niñas (31-46). Montevideo: Ediciones Universitarias.

Esquivel, V.(2013). El cuidado en loshogaresylas comunidades. Documento conceptual (Informes de investigación de OXFAM). Recuperado de https://oxfamilibrary. openrepository.com/bitstream/handle/10546/302287/rr-care-background-071013es.pdf;jsessionid=0BC9F95B58A26BAD01A2F3631107DAD9?sequence=2D/.

Flores, R. y Tena, O. (2014). Maternalismo y discursos feministas latinoamericanos sobre el trabajo de cuidados: un tejido en tensión. Íconos. Revista de Ciencias Sociales, (50), 27-42. Recuperado de https://www.researchgate. net/publication/287590871_Maternalismo_y_discursos_feministas_ latinoamericanos_sobre_el_trabajo_de_cuidados_un_tejido_en_tension.

Gilligan, C. (2013). La ética del cuidado. Cuadernos de la Fundación Víctor Grífols i Lucas (30). Recuperado de http://www.secpal.com/\%5CDocumentos\% 5CBlog\%5Ccuaderno30.pdf.

Grinberg, J. (2015). Entre la pediatría, el psicoanálisis y el derecho: apuntes sobre la recepción, reelaboración y difusión del 'maltrato infantil' en Argentina. Revista de Estudios Sociales, (53), 77-89. doi: 10.7440/res53.2015.06.

Instituto Nacional de Medicina Legal y Ciencias Forenses (INMLCF). (2017). Comportamiento de lesiones por violencia intrafamiliar: Colombia. Forensis 2017. Datos para la vida, 19(1), 171-254. Recuperado de https:// aprendiendoaserpapaz.redpapaz.org/wp-content/uploads/2018/08/Forensis2017-pdf-interactivo.compressed.pdf.

Izzedin, R. y Pachajoa, A. (2009). Pautas, prácticas y creencias acerca de crianza ayer y hoy. Liberabit. Revista de Psicología, 15(2), 109-115. Recuperado de http:// www.scielo.org.pe/pdf/liber/v15n2/a05v15n2.pdf.

Jaramillo, L. (2007). Concepción de infancia. Zona Próxima. Revista del Instituto de Estudios Superiores en Educación Universidad del Norte, (8), 108-123. Recuperado de https://www.academia.edu/37488080/Concepci\%C3\%B3n_ de_infancia.

Kempe, H., Silverman, F., Steele, B., Droegemueller, W., y Silver, H. (1962). The Battered-Child Syndrome. JAMA, 181(1), 17-24. doi: 10.1001/ jama.1962.03050270019004. 
El maltrato infantil: una aproximación socio-histórica a las prácticas de crianza y maternidad a la luz de las representaciones sociales

Lerner, G. (1993). The creation of feminist consciousness. New York, NY: Oxford University Press.

Martínez, C. (2015). Capítulo 2. Cambios demográficos. En Ministerio de Salud y Protección Social y Profamilia. Encuesta Nacional de Demografía y Salud (ENDS). Tomo I, 69-88. Bogotá D.C, Colombia: CTP All Solutions S. A. s.

Molina, M. (2006). Transformaciones histórico culturales del concepto de maternidad y sus repercusiones en la identidad de la mujer. Psykhe, 15(2), 93-103. doi: 10.4067/S0718-22282006000200009.

Mora, M. (2002). La teoría de las representaciones sociales de Serge Moscovici. Athenea Digital, (2), 1-25. Recuperado de https://www.raco.cat/index.php/ Athenea/article/viewFile/34106/33945.

Moscovici, S. (1979). El psicoanálisis, su imagen y su público. Buenos Aires, Argentina: Editorial Huemu.

O'Hagan, K. (1995). Emotional and psychological abuse: problems of definition. Child Abuse \& Neglect, 19(4), 449-461. doi: 10.1016/0145-2134(95)00006-t.

Organización Mundial de la Salud (oms). (2016). Maltrato infantil. Recuperado de https:/www.who.int/es/news-room/fact-sheets/detail/child-maltreatment.

Organización Panamericana de la Salud (OPS). (2017). Inspire. Siete estrategias para poner fin a la violencia contra los niños y las niñas. Recuperado de https://iris. paho.org/handle/10665.2/33741.

Palacios,J.,Moreno,M.yJiménez,J.(2014).Maltratoinfantil:Concepto, tipos,etiología. Infancia y Aprendizaje, 18(71), 7-21. doi: 10.1174/02103709560575442.

Palomar, C. (2005). Maternidad: Historia y Cultura. La ventana. Revista de Estudios de Género, 3(22), 35-67. Recuperado de http://www.scielo.org.mx/pdf/laven/ v3n22/1405-9436-laven-3-22-35.pdf.

Posada, A, Gómez, J. y Ramírez, H. (2008). Crianza humanizada: una estrategia para prevenir el maltrato infantil. Acta pediátrica de México, 29(5), 4553. Recuperado de https://academia.utp.edu.co/ps4/files/2016/09/Crianzahumanizada-una-estrategia-para-prevenir-el-maltrato-infantil-1-1.pdf.

Pulido, S., Castro-Osorio, J., Peña, M. y Ariza-Ramírez, D. (2013). Pautas, creencias y prácticas de crianza relacionadas con el castigo y su transmisión generacional. Revista latinoamericana de Ciencias Sociales, Niñez y Juventud, 11(1), 245-259. Recuperado de http://www.scielo.org.co/pdf/rlcs/v11n1/ v11n1a17.pdf. 
[22] Diana Marcela Bedoya-Gallego, Laura Carolina Jiménez Pérez, Maira Alejandra González-Gaviria, Jhon Anthony Careth Henao, Disney Tatiana Correa Cano y León Darío Valencia Arboleda

Rateau, P. y Lo Monaco, G. (2013). La Teoría de las Representaciones Sociales: orientaciones conceptuales, campos de aplicaciones y métodos. Revista CES Psicología, 6(1), 22-42. Recuperado de http://www.scielo.org.co/pdf/cesp/ v6n1/v6n1a03.pdf.

Racamier, P. (1979). De psychonalyse en psychiatrie. Etudes psychopathologiques. París: Payot.

Rodríguez-Escobar, G. y Rodríguez-Escobar, V. (2012). El maltrato infantil desde la perspectiva bioética. Revista Colombiana de Bioética, 7(2), 107-119. doi 10.18270/rcb.v7i2.1012.

Saavedra, E. y Castro, A. (2007). La investigación cualitativa, una discusión presente. Liberabit. Revista de Psicología, 13(13), 63-69. Recuperado de http://www. scielo.org.pe/pdf/liber/v13n13/a08v13n13.pdf.

Santana-Tavira, R., Sánchez-Ahedo, R. y Herrera-Basto, E. (1998). El maltrato infantil: un problema mundial. Salud Pública. México, 40(1), 58-65. Recuperado de https:// www.scielosp.org/article/ssm/content/raw/?resource_ssm_path=/media/assets/ spm/v40n1/Y0400109.pdf.

Santelices, M., Farkas, C., Montoya, M., Galleguillos, F., Carvacho, C., Fernández, A., Morales, L., Taboada, C. y Himmel, E. (2015). Factores predictivos de sensibilidad materna en infancia temprana. Psicoperspectivas, Individuo $y$ Sociedad. 14(1). 66-76. Recuperado de http://www.psicoperspectivas.cl/index. $\mathrm{php/psicoperspectivas/article/view/441.}$

Santiago, Z. (2007). Los niños en la historia. Los enfoques historiográficos de la infancia. Takwá. Revista de Historia, (11-12), 31b-50. Recuperado de http://148.202.18.157/sitios/publicacionesite/pperiod/takwa/Takwa1112/ zoila_santiago.pdf.

Sünker, H. y Moran-Ellis, J. (2018). Nuevos estudios de infancia, política de infancia y derechos de los niños y nińas. Sociedad e Infancias, (2), 171-188. Recuperado de https://dialnet.unirioja.es/servlet/articulo?codigo=6724740.

Tissera-Luna, M. (2018): El papel hegemónico de las organizaciones nogubernamentales y agencias internacionales en la conceptualización de la infancia. Sociedad e Infancias, (2), 39-57. Recuperado de https:/dialnet. unirioja.es/servlet/articulo? codigo $=6724740$.

Torres, N. (2006). Reflexiones acerca del desarrollo emocional de la madre, a partir de la obra de Winnicott. Persona, Revista de la Facultad de Psicología, (9), 203-2015. Recuperado de https://dialnet.unirioja.es/servlet/articulo?codigo=2872446. 
El maltrato infantil: una aproximación socio-histórica a las prácticas de crianza y maternidad a la luz de las representaciones sociales

Tronto, J. (1987). Más allá de la diferencia de género. Hacia una teoría del cuidado. Signs: Jornal of Women in Culture and Society, 12, 1-17. Recuperado de http:// www.unsam.edu.ar/escuelas/humanidades/centros/cedehu/material/(13)\%20 Texto\%20Joan\%20Tronto.pdf.

Urbina, J. y Ovalles, G. (2018). Teoría de las representaciones sociales. Una aproximación al estado del arte en América Latina. Psicogente, 21(40), 495-544. doi: $10.17081 /$ psico.21.40.3088.

Vindas, A. (2010). Reflexiones sobre el ejercicio actual de la maternidad. Revista Costarricense de Psicología, 29(43), 47-55. Recuperado de http://oaji.net/ articles/2016/3238-1464304915.pdf. 